\title{
Photo- and Collision-Induced Isomerization of a Charge-Tagged Norbornadiene-Quadricyclane System
}

Ugo Jacovella, Eduardo Carrascosa, Jack T. Buntine, Nicolai Ree, Kurt V. Mikkelsen, Martyn Jevric, Kasper Moth-Poulsen, and Evan J. Bieske*

Cite This: J. Phys. Chem. Lett. 2020, 11, 6045-6050

Read Online

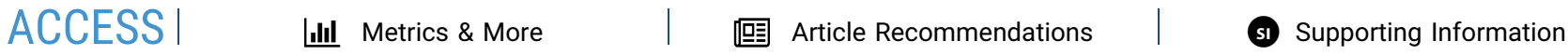

ABSTRACT: Molecular photoswitches based on the norbornadiene-quadricylane (NBD-QC) couple have been proposed as key elements of molecular solar thermal energy storage schemes. To characterize the intrinsic properties of such systems, reversible isomerization of a charge-tagged NBD-QC carboxylate couple is investigated in a tandem ion mobility mass spectrometer, using light to induce intramolecular $[2+2]$ cycloaddition of NBD carboxylate to form the QC carboxylate and driving the back reaction with molecular collisions. The NBD carboxylate photoisomerization action spectrum recorded by monitoring the QC carboxylate photoisomer extends from 290 to $360 \mathrm{~nm}$ with a maximum at $315 \mathrm{~nm}$, and in the longer wavelength region resembles the NBD carboxylate absorption spectrum recorded in solution. Key structural and photochemical properties of the NBD-QC carboxylate system, including the gas-phase absorption spectrum and the energy storage capacity, are determined through computational studies using density functional theory.

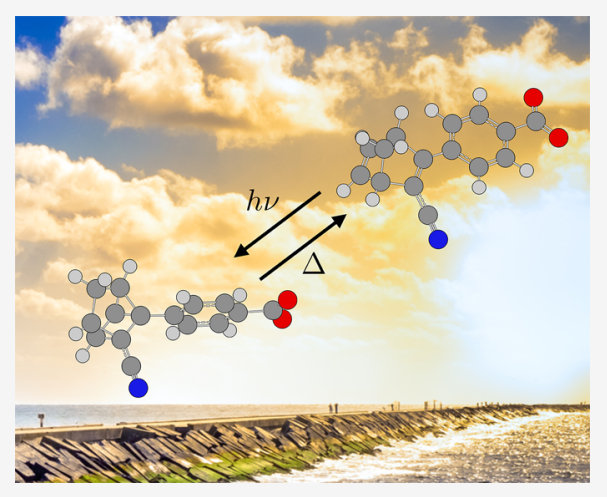

$\mathrm{E}$ ffective storage of harvested energy is essential for the widespread adoption of solar power. One proposed solution involves photochemical energy storage via isomerization reactions, whereby photochromic molecules are converted by solar radiation into metastable isomers with release of the stored energy achieved through either thermal, catalytic, electrochemical, or photochemical activation. ${ }^{1-5}$ This approach, known as a molecular solar thermal (MOST) system, $^{6}$ or solar thermal fuel system, ${ }^{7}$ has the potential to integrate the capture and storage of energy in a single molecular system.

Several photochromic motifs have been proposed for MOST systems including (fulvalene) tetracarbonyldiruthenium, ${ }^{8-10}$ azobenzene, ${ }^{11-14}$ dihydroazulene, ${ }^{15-17}$ and arguably the most studied system, norbornadiene (NBD)..$^{2,3,18-27} \mathrm{NBD}$ and its derivatives are appealing because of their high isomerization quantum yields for formation of the metastable quadricyclane (QC), low molecular weight, stability of the QC isomer over time, high fatigue resistance, and facile external triggering of the back-reaction from $\mathrm{QC}$ to NBD via thermal activation (see Figure 1).

A significant problem with the NBD chromophore is that its absorption onset lies in the ultraviolet $(<300 \mathrm{~nm})$, well away from the maximum of the solar radiation spectrum $(590 \mathrm{~nm})$. Although attempts have been made to shift the NBD absorption toward the maximum solar emission wavelength through derivatization, these changes compromise performance through the reduced half-life of the QC compound from 87000 years at room temperature for unsubstituted $\mathrm{QC}^{28}$ to a

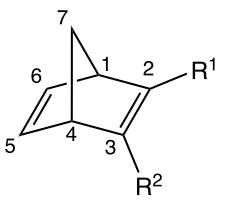

NBD

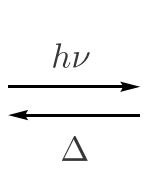

$\Delta$<smiles>CCC</smiles>

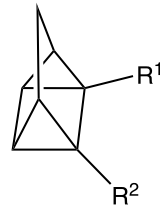

QC
Figure 1. Photoinduced isomerization of norbornadiene (NBD) to quadricyclane ( $\mathrm{QC})$ derivatives and the thermal back conversion.

few seconds for red-shifted variants. ${ }^{29}$ Recently, several approaches have increased the storage time of red-shifted QC molecules, ${ }^{24,26,27}$ although their efficiency requires further improvement for the systems to be viable for long-term energy storage applications.

A better understanding of the structures, relative stabilities, and isomerization dynamics of NBD and QC compounds is essential for the rational design of efficient NBD-QC MOST systems. Reliable quantum chemical calculations, such as those performed on the NBD/QC chromophores, ${ }^{30-33}$ should help guide the design of efficient MOST systems. Using single-

Received: April 19, 2020

Accepted: June 15, 2020

Published: June 15, 2020 
reference and multireference $a b$ initio methods it has been shown that, for substituted NBD-QC systems, the relevant photochemistry and thermal chemistry probably occur on the singlet surface through a $S_{0} / S_{1}$ conical intersection. ${ }^{31}$ Jorner and co-workers ${ }^{24}$ have modeled the performance of NBD-QC MOST systems in solution with the aid of $a b$ initio calculations, demonstrating that the quantum yield and thermal lifetime of QC derivatives increased with larger steric bulk at the C7 carbon (see Figure 1). However, these improvements were achieved at the expense of the energy storage capacities of the NBD-QC systems. Quant et al. ${ }^{34}$ have shown that solvent affects the absorption profile, kinetic stability, and photoisomerization dynamics of NBD-QC systems, emphasizing the challenges associated with benchmarking calculations for isolated molecules against experiments performed for molecules in solution.

In this work, we report gas-phase photochemical data for a charge-tagged NBD-QC system with photoisomerization of the NBD form to the $\mathrm{QC}$ form and back-conversion mediated by energetically controlled collisions with buffer gas. As shown in Figure 2 the target system is a charge-tagged NBD-QC

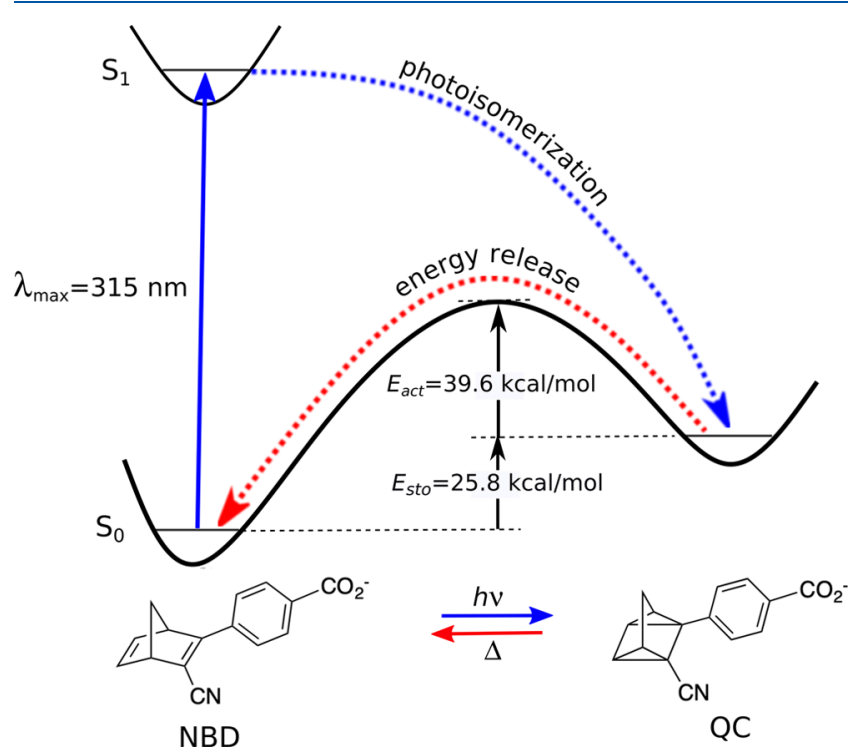

Figure 2. Photoisomerization of NBD carboxylate to QC carboxylate and the thermally induced back reaction. $\lambda_{\max } \Delta E_{\text {storage }}$ and $\Delta E_{\text {activation }}$ represent the maximum absorption wavelength, the storage energy of the NBD/QC system, and the activation energy for thermal backreaction, respectively. Calculations used to determine the thermodynamic quantities are described in the Supporting Information.

molecule with a cyano group conjugated through the olefin to a charged benzoate group. ${ }^{4}$ The cyano substituent shifts the absorption to longer wavelength, while the negatively charged carboxylate tag allows the photochemistry of the system to be probed in the gas phase with a sensitivity and specificity conferred by combining mass spectrometric techniques and laser excitation.

Interconversion of the NBD and QC carboxylates in the gas phase was investigated using a custom tandem ion mobility mass spectrometer (IMMS) shown in Figure 3 and described in refs 35-37. Briefly, NBD anions (deprotonated at the carboxylic group) were electrosprayed from a $10^{-5} \mathrm{~mol} \mathrm{~L}^{-1}$ methanolic solution and introduced as packets of ions into a drift region, where they were propelled by an electric field (44
$\mathrm{V} \mathrm{cm}^{-1}$ ) through $\mathrm{N}_{2}$ buffer gas ( $\approx 6$ Torr pressure). The NBD and $\mathrm{QC}$ isomers were separated temporally and spatially in the drift region according to their collision cross sections with the buffer gas and arrived at the detector as separated ion packets. By measuring the ion signal as a function of arrival time, it was possible to generate an arrival time distribution (ATD), which displayed distinct peaks associated with the NBD and QC isomers. Separation of the NBD and QC isomers was enhanced by addition to the buffer gas of $\approx 1 \%$ propan-2-ol, a commonly used mobility modifier. ${ }^{38,39}$

For the gas-phase isomerization measurements, the IMMS was used in tandem mode, with a pulsed electrostatic ion gate situated midway along the drift region selecting the target isomer ions, which were excited immediately after the gate either by a 5 ns pulse of light from an optical parametric oscillator (OPO, EKSPLA NT342B) or through energetic collisions with buffer gas molecules in a short $3 \mathrm{~mm}$ collision zone (slammer) where the electric field could be varied. ${ }^{37}$ Resulting isomers were separated in the second stage of the drift region. The photoisomerization measurements were run at $40 \mathrm{~Hz}$ with alternate ion packets exposed to the output of the pulsed OPO (operating at $20 \mathrm{~Hz}$ ), so that laser-on and laser-off ATDs were recorded, the difference between which revealed the effect of light on the drifting ions. The light fluence was $\approx 0.2\left(\mathrm{~mJ} / \mathrm{cm}^{2}\right) /$ pulse, with conversion of $<5 \%$ of the NBD to QC at the wavelength of maximum response (315 $\mathrm{nm})$.

It is worth noting that the peak in the ATD for an isomer formed through photoexcitation or collisional activation between the two drift regions appears between the peaks for the original isomer and final isomer for an ATD recorded when both isomers passed through the entire drift region (first and second drift regions). This is because the ions drift through the first drift region as the original isomer and then through the second drift region as the isomer formed through excitation by light or collisions. If the first and second drift regions had the same length and applied electric field, the photoisomer peak would appear midway between the peaks for the two isomers if they had separated traveling through both drift regions. However, the second drift region is longer and includes an ion funnel (IF2) where the electric field is lower. Therefore, the ions travel for further as the product isomer than as the original isomer, displacing the peak from the midpoint.

As shown in Figure 4a, the ATD peak at $18.0 \mathrm{~ms}$ is assigned to the more stable NBD carboxylate isomer, whereas the peak at $18.3 \mathrm{~ms}$, which appeared after the solution in the electrospray syringe was exposed to $315 \mathrm{~nm}$ light, is assigned to the less stable QC carboxylate isomer, which lies $25.8 \mathrm{kcal} /$ mol higher in energy (see the Supporting Information for calculation details). The relative intensities of the NBD and QC ATD peaks depended on the amplitude of the RF voltage driving the first ion funnel (IF1). High RF voltages, which promote energetic collisions, reduced the relative intensity of the QC peak, consistent with thermal QC $\rightarrow$ NBD conversion.

Exposing the drifting NBD carboxylate ions to light with a wavelength over the $290-350 \mathrm{~nm}$ range resulted in the appearance of a new ATD peak with an arrival time expected for QC carboxylate (see Figure 4b). This constitutes evidence that in the gas-phase NBD carboxylate can undergo a photoinduced $[2+2]$ cycloaddition to its valence $\mathrm{QC}$ isomer. Loss of the NBD carboxylate is balanced by formation of the QC carboxylate, indicating that other loss mechanisms such as photodetachment and photodissociation play a minor role in 

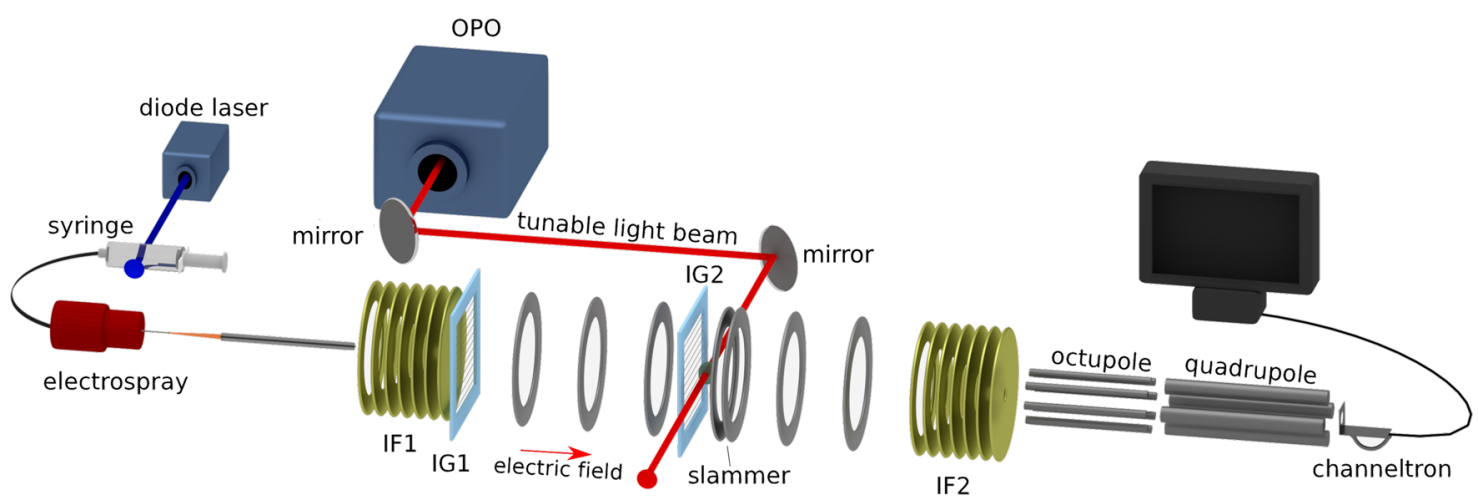

Figure 3. Schematic view of the tandem ion mobility mass spectrometer (IMMS). Ions generated by electrospray ionization were collected by an ion funnel (IF1), before being launched as a pulse through an electrostatic ion gate (IG1) into the drift region where they were propelled through $\mathrm{N}_{2}$ buffer gas by an electric field of $44 \mathrm{~V} \mathrm{~cm}^{-1}$. In the drift region, the isomer ions separated according to their collision cross sections with $\mathrm{N}_{2}$ buffer gas. Target isomers were selected using an electrostatic ion gate (IG2), before being exposed to tunable light or energetic collisions in the slammer region. Resulting isomer ions passed through the second part of the drift region were collected by an ion funnel (IF2), before passing through an octopole ion guide and quadrupole mass filter and finally reaching a channeltron detector connected to a multichannel scaler.

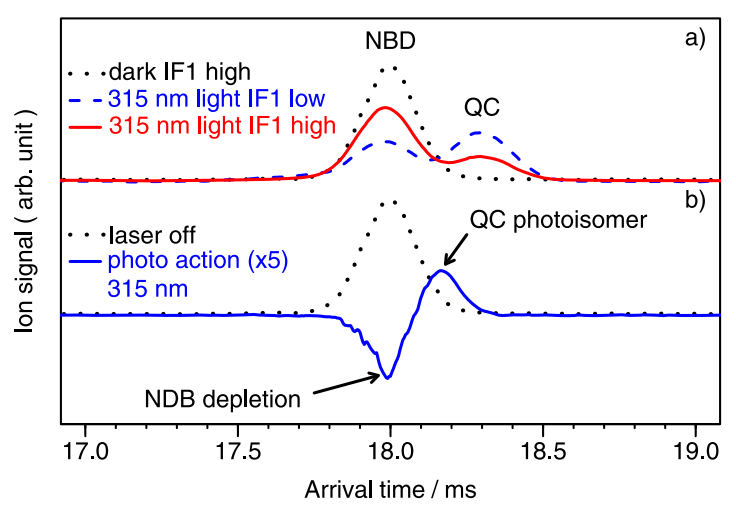

Figure 4. (a) Arrival time distributions (ATDs) for the NBD-QC carboxylate system obtained using $\mathrm{N}_{2}$ buffer gas seeded with $\approx 1 \%$ propan-2-ol. ATDs are shown for a solution protected from light (black dotted trace) and after exposure of the solution to $315 \mathrm{~nm}$ light for low (blue dotted trace) and high (red solid trace) RF drive voltage to the first ion funnel (IF1). (b) Laser-off ATD (black dotted trace) and laser-on/laser-off difference ATD (blue solid trace) for NBD exposed to $315 \mathrm{~nm}$ light in the tandem IMMS. Note that the QC photoisomer peak in (b) appears between the NBD and QC peaks in (a), as the ions pass through the first drift region stage as NBD and the second drift region stage as QC (see text).

the drift tube over this wavelength range. The absence of photodetachment, at least down to $315 \mathrm{~nm}$, is perhaps surprising given that the calculated adiabatic electron affinities of NBD carboxylate and QC caboxylate are 3.84 and $3.70 \mathrm{eV}$, respectively (corresponding to wavelengths of 322 and 335 $\mathrm{nm}$; Table 1). In both cases, direct electron detachment is presumably not appreciable at the light fluences used $(\approx 0.2$ $\left(\mathrm{mJ} / \mathrm{cm}^{2}\right) /$ pulse $)$. Furthermore, although electronically excited NBD ions that undergo rapid nonradiative decay may give NBD or QC ions with sufficient vibrational energy to undergo vibrational autodetachment, these ions are likely to be collisionally quenched in the drift region, where the collision rate is $10^{8}-10^{9} \mathrm{~s}^{-1}$, before autodetachment can occur.

The photoisomerization action (PISA) spectrum for the NBD carboxylate isomer was obtained by monitoring the QC carboxylate isomer yield as a function of OPO wavelength. As shown in Figure 5, the band maxima of the NBD $\rightarrow$ QC PISA spectrum agrees with the absorption spectrum calculated using
Table 1. Comparison of the Experimental Absorption Onset $\left(\lambda_{\text {onset }}\right)$ and Band Maximum $\left(\lambda_{\max }\right)$ with the Calculated Band Maximum $\left(\lambda_{\text {calc }}\right)$ and Adiabatic Electron Affinity (AEA) ${ }^{a}$

$\begin{array}{lcccc} & \lambda_{\text {onset }} / \mathrm{nm} & \lambda_{\text {max }} / \mathrm{nm} & \lambda_{\text {calc }} / \mathrm{nm} & \mathrm{AEA} / \mathrm{eV} \\ \mathrm{NBD}_{\text {(gas) }} & 355 & 315 & 317 & 3.84 \\ \mathrm{NBD}_{\text {(sol) }} & 355 & 315 & 303 & \\ \mathrm{QC}_{\text {(gas) }} & & & 220 & 3.70 \\ \mathrm{QC}_{\text {(sol) }} & 296 & 256 & 232 & \end{array}$

${ }^{a}$ Computational details can be found in the Supporting Information. Results are given for NBD and QC carboxylate in gas phase (gas) and in methanolic solution (sol).

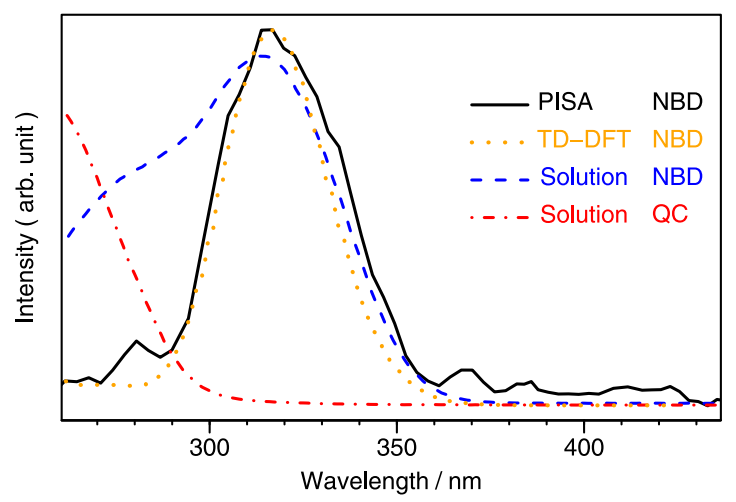

Figure 5. PISA spectrum corresponding to the QC carboxylate ion yield from the NBD carboxylate as a function of the wavelength (solid black trace) and calculated TD-DFT absorption spectrum of NBD carboxylate in vacuum (dotted orange trace). The electronic absorption spectra of QC and NBD carboxylates in methanolic solution are shown as red dot-dash and blue dashed traces, respectively.

TD-DFT (CAM-B3LYP/6-311+G(d)level of theory) consistent with the photoisomerization quantum yield being independent of wavelength and with a barrierless access to a conical intersection on the excited state potential energy surface of the NBD carboxylate ${ }^{33}$ (represented in Figure 2).

The NBD carboxylate PISA spectrum is compared to the absorption spectra of NBD carboxylate and QC carboxylate in methanolic solution in Figure 5. The absorption spectrum of the initial sample in solution, shown as dashed blue trace in 
Figure 5, is associated with the more stable NBD isomer. The absorption spectrum of the QC form shown in Figure 5 was obtained by exposing the cuvette to $315 \mathrm{~nm}$ light prior to the measurement. The PISA and solution absorption spectra of NBD carboxylate both exhibit a prominent peak with an onset at $350 \mathrm{~nm}$ and maximum at around $315 \mathrm{~nm}$. However, the absorption spectrum of NBD carboxylate in solution exhibits a shoulder to higher energy that is absent from the PISA spectrum. According to the calculated electronic absorption spectrum presented in the Supporting Information, the transition responsible for this shoulder originates from methanol-NBD carboxylate interactions.

The first dipole-allowed transition of the QC carboxylate is predicted to occur at $220 \mathrm{~nm}$ (see Table 1), outside the operating wavelength range of the OPO used. Although it was not possible to photoisomerize the QC carboxylate, energetic buffer gas collisions promoted $\mathrm{QC} \rightarrow \mathrm{NBD}$ isomerization, as shown in Figure 6a, where the ion signal is plotted as a
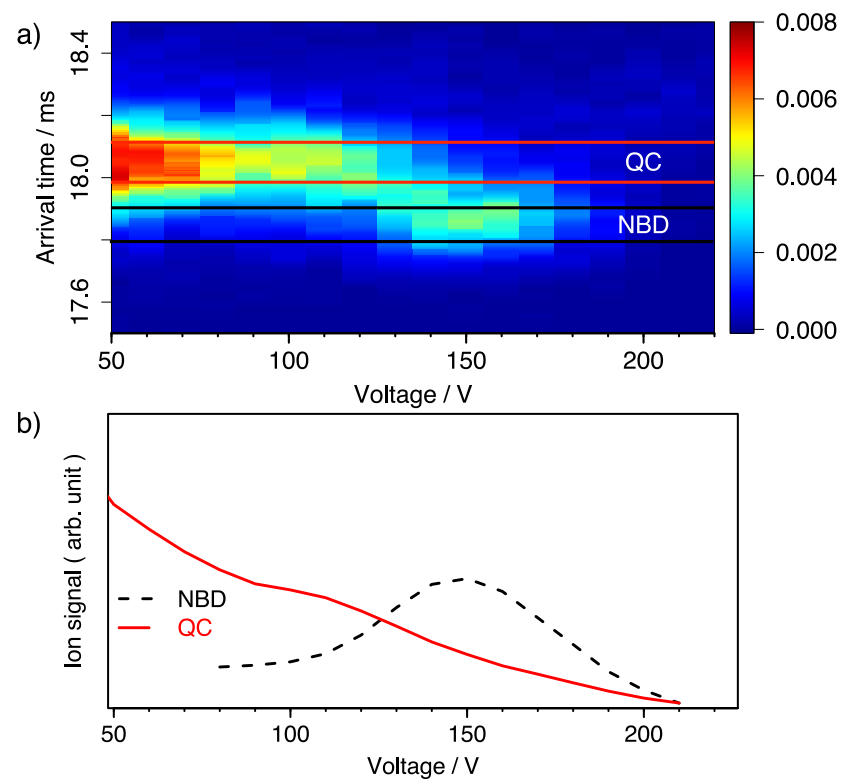

Figure 6. Transformation of the QC carboxylate isomer to the NBD carboxylate isomer by collisional activation with $\mathrm{N}_{2}$ buffer gas. (a) Ion signal as a function of slammer voltage and arrival time. The two sets of horizontal lines indicate the arrival time ranges for NBD and QC carboxylates. Note that the separation between the NBD and QC arrival times is reduced compared to Figure 4a because the NBD ions are formed from QC ions between the first and second drift regions. Arrival times for all species are less than in Figure 4 because of the high electric field in the slammer region. (b) NBD and QC carboxylate ion signal (dashed black and solid red, respectively), as a function of slammer voltage.

function of the voltage applied between the slammer electrodes and ion arrival time. The QC ions arrive at $18.05 \mathrm{~ms}$, whereas the more compact NBD ions arrive at $17.85 \mathrm{~ms}$. As shown in Figure $6 \mathrm{~b}$, where the NBD and QC carboxylate ion signals are plotted as a function of slammer voltage, with increasing collision energy there is an overall loss of ions due to fragmentation but clearly also conversion of the QC carboxylate to NBD carboxylate via collisional activation with a threshold at $\approx 100 \mathrm{~V}$. Collisions presumably provide sufficient energy to surmount the $\mathrm{QC} \rightarrow \mathrm{NBD}$ activation barrier calculated to be $\Delta E_{\text {activation }}=39.6 \mathrm{kcal} / \mathrm{mol}$ (see the Supporting
Information for details). The collision induced conversion of NBD to QC, is consistent with the increase in the relative proportion of NBD compared to QC with amplitude of the RF drive voltage for the first ion funnel (IF1) (see Figure 4a).

The current work augments early gas-phase photochemical studies of unsubstituted neutral NBD, which features an absorption spectrum with an onset at $270 \mathrm{~nm}$, a shoulder at $230 \mathrm{~nm}$, and several sharper bands between 226 and 199 nm. ${ }^{40,41}$ Upon exposure to $253.7 \mathrm{~nm}$ light, unsubstituted neutral NBD decomposes to cyclopentadiene and acetylene with isomerization to toluene being a minor channel. ${ }^{40,41}$ The absence of fragmentation for the NBD carboxylate system following excitation in the $315 \mathrm{~nm}$ region is likely due to the longer excitation wavelength, and the fact that the excited molecules are deactivated through rapid collisions with $\mathrm{N}_{2}$ buffer gas molecules in the drift region where the collision rate is $10^{8}-10^{9} \mathrm{~s}^{-1}$.

To summarize, we have demonstrated reversible isomerization of an isolated NBD-QC system with NBD $\rightarrow$ QC isomerization through photoinduced $[2+2]$ cycloaddition, and the reverse $\mathrm{QC} \rightarrow \mathrm{NBD}$ reaction promoted through collisional activation. The NBD $\rightarrow$ QC photoisomerization action spectrum has a maximum at $315 \mathrm{~nm}$, matching the calculated vertical electronic absorption, and maximum of the absorption spectrum for NBD dissolved in methanol. Agreement between the PISA spectrum, the solution absorption spectrum, and the calculated absorption spectrum is consistent with a barrierless photoisomerizaton on the excited state potential energy surface of the NBD carboxylate. Overall, we have shown that a tandem ion mobility mass spectrometer is a suitable platform to study the isomerization of charge-tagged NBD-QC and related systems, yielding data that should serve to benchmark state-of-the-art quantum chemical calculations, and ultimately help optimize the performance of molecular solar thermal storage systems. Hopefully, this investigation will pave the way for combined studies of other substituted NBDQC systems in the gas phase, in solution, and computationally, in order to test whether the minor effect of solvent on the absorption spectrum in the longer wavelength region is a general phenomenon, and if the TD-DFT CAM B3LYP methodology is appropriate for predicting spectra of a broader range of NBD-QC systems. In addition, it would be interesting to study the thermal $\mathrm{QC} \rightarrow \mathrm{NBD}$ isomerization for a range of substituted NBD-QC systems in a temperaturecontrolled ion trap or drift tube to explore the effect of substituents on the isomerization barrier.

\section{ASSOCIATED CONTENT}

\section{Supporting Information}

The Supporting Information is available free of charge at https://pubs.acs.org/doi/10.1021/acs.jpclett.0c01198.

Additional material describing the calculated electronic absorption spectra, XYZ coordinates of the molecular geometries with corresponding energies, and IRC calculations, UV-vis spectra and simulations (PDF)

\section{AUTHOR INFORMATION}

\section{Corresponding Author}

Evan J. Bieske - School of Chemistry, The University of Melbourne, Victoria 3010, Australia; 이이이.org/0000-00031848-507X; Email: evanjb@unimelb.edu.au 


\section{Authors}

Ugo Jacovella - School of Chemistry, The University of Melbourne, Victoria 3010, Australia

Eduardo Carrascosa - School of Chemistry, The University of Melbourne, Victoria 3010, Australia; (1) orcid.org/0000-00034338-8669

Jack T. Buntine - School of Chemistry, The University of Melbourne, Victoria 3010, Australia

Nicolai Ree - Department of Chemistry, University of Copenhagen, 2100 Copenhagen Ø, Denmark

Kurt V. Mikkelsen - Department of Chemistry, University of Copenhagen, 2100 Copenhagen Ø, Denmark; 이이.org/ 0000-0003-4090-7697

Martyn Jevric - Department of Chemistry and Chemical Engineering, Chalmers University of Technology, 41296 Gothenburg, Sweden

Kasper Moth-Poulsen - Department of Chemistry and Chemical Engineering, Chalmers University of Technology,

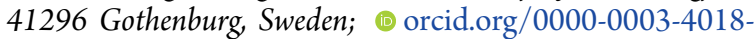
4927

Complete contact information is available at:

https://pubs.acs.org/10.1021/acs.jpclett.0c01198

\section{Notes}

The authors declare no competing financial interest.

\section{ACKNOWLEDGMENTS}

This work was supported by the Australian Research Council Discovery Project (DP150101427, DP160100474) and Future Fellowship (FT130101304) schemes. U.J. acknowledges support from the Swiss National Science Foundation (P2EZP2 178429). E.C. acknowledges support by the Austrian Science Fund (FWF) through a Schrödinger Fellowship (No. J4013-N36). K.V.M. thanks the Center for Exploitation of Solar Energy for support. N.R. thanks H. C. Ørsted Selskabet and Ørsted A/S for financial support in terms of the Ørsted Scholarship 2018. M.J. and K.M.P. acknowledge support from the Knut and Alice Wallenberg Foundation and Swedish Strategic Research Foundation (SSF).

\section{REFERENCES}

(1) Wentworth, W.; Chen, E. Simple thermal decomposition reactions for storage of solar thermal energy. Sol. Energy 1976, 18, 205-214.

(2) Jones, G. Cycloaddition reactions involving 4 n electrons: $(2+2)$ cycloaddition; photochemical energy storage systems based on reversible valence photoisomerization. Studies in Organic Chemistry 1990, 40, 514-538.

(3) Dreos, A.; Wang, Z.; Udmark, J.; Ström, A.; Erhart, P.; Börjesson, K.; Brøndsted Nielsen, M.; Moth-Poulsen, K. Liquid norbornadiene photoswitches for solar energy storage. Adv. Energy Mater. 2018, 8, 1703401.

(4) Schuschke, C.; Hohner, C.; Jevric, M.; Petersen, A. U.; Wang, Z.; Schwarz, M.; Kettner, M.; Waidhas, F.; Fromm, L.; Sumby, C. J.; et al. Solar energy storage at an atomically defined organic-oxide hybrid interface. Nat. Commun. 2019, 10, 2384.

(5) Waidhas, F.; Jevric, M.; Bosch, M.; Yang, T.; Franz, E.; Liu, Z.; Bachmann, J.; Moth-Poulsen, K.; Brummel, O.; Libuda, J. Electrochemically controlled energy release from a norbornadiene-based solar thermal fuel: increasing the reversibility to $99.8 \%$ using HOPG as the electrode material. J. Mater. Chem. A 2020, DOI: 10.1039/ D0TA00377H.

(6) Moth-Poulsen, K.; Ćoso, D.; Börjesson, K.; Vinokurov, N.; Meier, S. K.; Majumdar, A.; Vollhardt, K. P. C.; Segalman, R. A.
Molecular solar thermal (MOST) energy storage and release system. Energy Environ. Sci. 2012, 5, 8534-8537.

(7) Kucharski, T. J.; Ferralis, N.; Kolpak, A. M.; Zheng, J. O.; Nocera, D. G.; Grossman, J. C. Templated assembly of photoswitches significantly increases the energy-storage capacity of solar thermal fuels. Nat. Chem. 2014, 6, 441-447.

(8) Boese, R.; Cammack, J. K.; Matzger, A. J.; Pflug, K.; Tolman, W. B.; Vollhardt, K. P. C.; Weidman, T. W. Photochemistry of (fulvalene)tetracarbonyldiruthenium and its derivatives: Efficient light energy storage devices. J. Am. Chem. Soc. 1997, 119, 6757-6773.

(9) Kanai, Y.; Srinivasan, V.; Meier, S. K.; Vollhardt, K. P. C.; Grossman, J. C. Mechanism of thermal reversal of the (fulvalene)tetracarbonyldiruthenium photoisomerization: toward molecular solar-thermal energy storage. Angew. Chem., Int. Ed. 2010, 49, $8926-8929$.

(10) Börjesson, K.; Ćoso, D.; Gray, V.; Grossman, J. C.; Guan, J.; Harris, C. B.; Hertkorn, N.; Hou, Z.; Kanai, Y.; Lee, D.; et al. Exploring the potential of fulvalene dimetals as platforms for molecular solar thermal energy storage: Computations, syntheses, structures, kinetics, and catalysis. Chem. - Eur. J. 2014, 20, 1558715604.

(11) Feng, Y.; Liu, H.; Luo, W.; Liu, E.; Zhao, N.; Yoshino, K.; Feng, W. Covalent functionalization of graphene by azobenzene with molecular hydrogen bonds for long-term solar thermal storage. Sci. Rep. 2013, 3, 3260.

(12) Masutani, K.; Morikawa, M.; Kimizuka, N. A liquid azobenzene derivative as a solvent-free solar thermal fuel. Chem. Commun. 2014, 50, 15803-15806.

(13) Han, G. D.; Park, S. S.; Liu, Y.; Zhitomirsky, D.; Cho, E.; Dincă, M.; Grossman, J. C. Photon energy storage materials with high energy densities based on diacetylene-azobenzene derivatives. $J$. Mater. Chem. A 2016, 4, 16157-16165.

(14) Cho, E. N.; Zhitomirsky, D.; Han, G. G. D.; Liu, Y.; Grossman, J. C. Molecularly engineered azobenzene derivatives for high energy density solid-state solar thermal fuels. ACS Appl. Mater. Interfaces 2017, 9, 8679-8687.

(15) Skov, A. B.; Broman, S. L.; Gertsen, A. S.; Elm, J.; Jevric, M.; Cacciarini, M.; Kadziola, A.; Mikkelsen, K. V.; Brøndsted Nielsen, M. Aromaticity-controlled energy storage capacity of the dihydroazulenevinylheptafulvene photochromic system. Chem. - Eur. J. 2016, 22, $14567-14575$.

(16) Wang, Z.; Udmark, J.; Börjesson, K.; Rodrigues, R.; Roffey, A.; Abrahamsson, M.; Brøndsted Nielsen, M.; Moth-Poulsen, K. Evaluating dihydroazulene/vinylheptafulvene photoswitches for solar energy storage applications. ChemSusChem 2017, 10, 3049-3055.

(17) Nielsen, M B; Ree, N; Mikkelsen, K V; Cacciarini, M Tuning the dihydroazulene/vinylheptafulvene couple for storage of solar energy. Russ. Chem. Rev. 2020, 89, 573.

(18) Yoshida, Z. New molecular energy storage systems. J. Photochem. 1985, 29, 27-40.

(19) Bren', V A; Dubonosov, A. D; Minkin, V. I; Chernoivanov, V A Norbornadiene-quadricyclane an effective molecular system for the storage of solar energy. Russ. Chem. Rev. 1991, 60, 451-469.

(20) Dubonosov, A. D.; Bren, V. A.; Chernoivanov, V. A. Norbornadiene-quadricyclane as an abiotic system for the storage of solar energy. Russ. Chem. Rev. 2002, 71, 917.

(21) Gray, V.; Lennartson, A.; Ratanalert, P.; Börjesson, K.; MothPoulsen, K. Diaryl-substituted norbornadienes with red-shifted absorption for molecular solar thermal energy storage. Chem. Commun. 2014, 50, 5330-5332.

(22) Quant, M.; Lennartson, A.; Dreos, A.; Kuisma, M.; Erhart, P.; Börjesson, K.; Moth-Poulsen, K. Low molecular weight norbornadiene derivatives for molecular solar-thermal energy storage. Chem. Eur. J. 2016, 22, 13265-13274.

(23) Brummel, O.; Besold, D.; Döpper, T.; Wu, Y.; Bochmann, S.; Lazzari, F.; Waidhas, F.; Bauer, U.; Bachmann, P.; Papp, C.; et al. Energy storage in strained organic molecules: (Spectro)electrochemical characterization of norbornadiene and quadricyclane. ChemSusChem 2016, 9, 1424-1432. 
(24) Jorner, K.; Dreos, A.; Emanuelsson, R.; El Bakouri, O.; Fdez. Galván, I.; Börjesson, K.; Feixas, F.; Lindh, R.; Zietz, B.; MothPoulsen, K.; et al. Unraveling factors leading to efficient norbornadiene-quadricyclane molecular solar-thermal energy storage systems. J. Mater. Chem. A 2017, 5, 12369-12378.

(25) Dreos, A.; Börjesson, K.; Wang, Z.; Roffey, A.; Norwood, Z.; Kushnir, D.; Moth-Poulsen, K. Exploring the potential of a hybrid device combining solar water heating and molecular solar thermal energy storage. Energy Environ. Sci. 2017, 10, 728-734.

(26) Jevric, M.; Petersen, A. U.; Mansø, M.; Kumar Singh, S.; Wang, Z.; Dreos, A.; Sumby, C.; Brøndsted Nielsen, M.; Börjesson, K.; Erhart, P.; et al. Norbornadiene-based photoswitches with exceptional combination of solar spectrum match and long-term energy storage. Chem. - Eur. J. 2018, 24, 12767-12772.

(27) Mansø, M.; Petersen, A. U.; Wang, Z.; Erhart, P.; Brøndsted Nielsen, M.; Moth-Poulsen, K. Molecular solar thermal energy storage in photoswitch oligomers increases energy densities and storage times. Nat. Commun. 2018, 9, 1945.

(28) Kabakoff, D. S.; Buenzli, J. C.; Oth, J. F.; Hammond, W. B.; Berson, J. A. Enthalpy and kinetics of isomerization of quadricyclane to norbornadiene. Strain energy of quadricyclane. J. Am. Chem. Soc. 1975, 97, 1510-1512.

(29) Miki, S.; Asako, Y.; Yoshida, Z. Photochromic solid films prepared by doping with donor-acceptor norbornadienes. Chem. Lett. 1987, 16, 195-198.

(30) Antol, I. Photodeactivation paths in norbornadiene. J. Comput. Chem. 2013, 34, 1439-1445.

(31) Kuisma, M. J.; Lundin, A. M.; Moth-Poulsen, K.; Hyldgaard, P.; Erhart, P. Comparative ab-initio study of substituted norbornadienequadricyclane compounds for solar thermal storage. J. Phys. Chem. C 2016, 120, 3635-3645.

(32) Kuisma, M.; Lundin, A.; Moth-Poulsen, K.; Hyldgaard, P.; Erhart, P. Optimization of norbornadiene compounds for solar thermal storage by first-principles calculations. ChemSusChem 2016, 9 , $1786-1794$

(33) Nucci, M.; Marazzi, M.; Frutos, L. M. Mechanochemical improvement of norbornadiene-based molecular solar-thermal systems performance. ACS Sustainable Chem. Eng. 2019, 7, 1949619504.

(34) Quant, M.; Hamrin, A.; Lennartson, A.; Erhart, P.; MothPoulsen, K. Solvent effects on the absorption profile, kinetic stability, and photoisomerization process of the norbornadiene-quadricyclanes system. J. Phys. Chem. C 2019, 123, 7081-7087.

(35) Adamson, B. D.; Coughlan, N. J. A.; Continetti, R. E.; Bieske, E. $\mathrm{J}$. Changing the shape of molecular ions: photoisomerization action spectroscopy in the gas phase. Phys. Chem. Chem. Phys. 2013, 15, 9540-9548.

(36) Adamson, B. D.; Coughlan, N. J. A.; Markworth, P. B.; Continetti, R. E.; Bieske, E. J. An ion mobility mass spectrometer for investigating photoisomerization and photodissociation of molecular ions. Rev. Sci. Instrum. 2014, 85, 123109.

(37) Coughlan, N. J. A.; Scholz, M. S.; Hansen, C. S.; Trevitt, A. J.; Adamson, B. D.; Bieske, E. J. Photo and collision induced isomerization of a cyclic retinal derivative: an ion mobility study. J. Am. Soc. Mass Spectrom. 2016, 27, 1483-1490.

(38) Rorrer, L. C.; Yost, R. A. Solvent vapor effects in planar highfield asymmetric waveform ion mobility spectrometry: Solvent trends and temperature effects. Int. J. Mass Spectrom. 2015, 378, 336-346.

(39) Waraksa, E.; Perycz, U.; Namieśnik, J.; Sillanpää, M.; Dymerski, T.; Wójtowicz, M.; Puton, J. Dopants and gas modifiers in ion mobility spectrometry. TrAC, Trends Anal. Chem. 2016, 82, 237-249.

(40) Roquitte, B. Photolysis of $\Delta^{2,5}$-bicyclo [2.2.1] heptadiene in the vapor phase. J. Am. Chem. Soc. 1963, 85, 3700.

(41) Roquitte, B. The photochemistry of 2,5-bicyclo [2.2.1] heptadiene1. J. Phys. Chem. 1965, 69, 2475. 


\section{University Library}

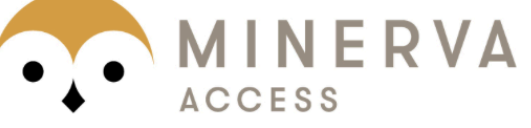

A gateway to Melbourne's research publications

Minerva Access is the Institutional Repository of The University of Melbourne

Author/s:

Jacovella, U;Carrascosa, E;Buntine, JT;Ree, N;Mikkelsen, K;Jevric, M;Moth-Poulsen, $\mathrm{K}$;Bieske, EJ

Title:

Photo- and Collision-Induced Isomerization of a Charge-Tagged NorbornadieneQuadricyclane System

Date:

2020-08-06

Citation:

Jacovella, U., Carrascosa, E., Buntine, J. T., Ree, N., Mikkelsen, K., Jevric, M., MothPoulsen, K. \& Bieske, E. J. (2020). Photo- and Collision-Induced Isomerization of a ChargeTagged Norbornadiene-Quadricyclane System. JOURNAL OF PHYSICAL CHEMISTRY LETTERS, 11 (15), pp.6045-6050. https://doi.org/10.1021/acs.jpclett.0c01198.

Persistent Link:

http://hdl.handle.net/11343/252489

License:

cc-by 